\title{
Metalaxyl Resistance in Phytophthora infestans: Assessing Role of RPA190 Gene and Diversity Within Clonal Lineages
}

\author{
Michael E. H. Matson, Ian M. Small, William E. Fry, and Howard S. Judelson
}

First and fourth authors: Department of Plant Pathology and Microbiology, University of California, Riverside, CA 92521; and second and third authors: Section of Plant Pathology and Plant-Microbe Biology, Cornell University, Ithaca, NY 14853. Accepted for publication 27 July 2015.

\begin{abstract}
Matson, M. E. H., Small, I. M., Fry, W. E., and Judelson, H. S. 2015. Metalaxyl resistance in Phytophthora infestans: Assessing role of RPA190 gene and diversity within clonal lineages. Phytopathology 105:1594-1600.

Prior work has shown that the inheritance of resistance to metalaxyl, an oomycete-specific fungicide, is complex and may involve multiple genes. Recent research indicated that a single nucleotide polymorphism (SNP) in the gene encoding RPA190, the largest subunit of RNA polymerase I, confers resistance to metalaxyl (or mefenoxam) in some isolates of the potato late blight pathogen Phytophthora infestans. Using both DNA sequencing and high resolution melt assays for distinguishing RPA190

alleles, we show here that the SNP is absent from certain resistant isolates of $P$. infestans from North America, Europe, and Mexico. The SNP is present in some members of the US-23 and US-24 clonal lineages, but these tend to be fairly sensitive to the fungicide based on artificial media and field test data. Diversity in the level of sensitivity, RPA190 genotype, and RPA190 copy number was observed in these lineages but were uncorrelated. Controlled laboratory crosses demonstrated that RPA190 did not cosegregate with metalaxyl resistance from a Mexican and British isolate. We conclude that while metalaxyl may be used to control many contemporary strains of $P$. infestans, an assay based on RPA190 will not be sufficient to diagnose the sensitivity levels of isolates.
\end{abstract}

The development of fungicide resistance is a challenge in many pathosystems. When the genetic basis of resistance is known, DNAbased diagnostics hold the potential of predicting whether an outbreak is controllable by the fungicide. This strategy has become common for Qo inhibitors, where most cases of resistance are caused by a single point mutation in the cytochrome $b$ gene (Dufour et al. 2011; Grasso et al. 2006). The approach becomes more complex when multiple genes are involved. With sterol biosynthesis inhibitors, for example, reduced sensitivity is known to result from changes in the structure and/or expression of at least three genes (Omrane et al. 2015; Proffer et al. 2006).

The phenylamide compound metalaxyl was introduced in the 1970s as a systemic fungicide against oomycetes and has been used widely against Phytophthora, Pythium, and downy mildews. Strains of these pathogens with resistance to metalaxyl (including formulations with mefenoxam, the active R-isomer) have since emerged in many locations (Davidse et al. 1981; Diriwachter et al. 1987; Taylor et al. 2002). For example, starting in the 1980s in Europe and in North America in the 1990s, many strains of the potato late blight pathogen Phytophthora infestans sampled from outbreaks were resistant to metalaxyl. In the United States, the most prevalent resistant genotypes were assigned to asexual lineages called US-6, 7, 8, and 11 based on allozyme and DNA fingerprints (Goodwin et al. 1998). In 2009, populations of $P$. infestans in many parts of the United States underwent another major change as the prior genotypes were displaced by lineages named US-22, 23, and 24 ( $\mathrm{Hu}$ et al. 2012). These have been reported to lack strong resistance to metalaxyl based on assays on artificial media. US-23 is reported to be fairly sensitive and US-24 to have intermediate sensitivity, compared with resistant lineages like US-8 and 11 (Hu

Corresponding author: H. S. Judelson; E-mail address: howard.judelson@ucr.edu

*The $\boldsymbol{e}$-Xtra logo stands for "electronic extra" and indicates that one supplementary table is published online.

http://dx.doi.org/10.1094/PHYTO-05-15-0129-R

(C) 2015 The American Phytopathological Society et al. 2012). Since US-23 now dominates many parts of the country, particularly the East and Midwest, metalaxyl has potential value for controlling late blight.

Genetic analyses of the inheritance of metalaxyl insensitivity in P. infestans isolates from North America, Mexico, and Europe showed that resistance in each strain was determined by a major semidominant locus (Fabritius et al. 1997; Judelson and Roberts 1999; Lee et al. 1999; Shattock 1988). The data also supported the influence of minor modifying loci, as would be seen for a quantitative trait. Strains thus can exhibit a range of sensitivities depending on zygosity at the major locus and the minor genes. Similar conclusions were drawn from studies of a downy mildew (Crute and Harrison 1988).

Biochemical studies in Phytophthora found that metalaxyl inhibits the synthesis of ribosomal RNA, suggesting that it may target RNA polymerase I (Davidse et al. 1988). Pursuing that lead, Randall et al. (2014) associated resistance with a single nucleotide polymorphism, SNP T1145A, in the gene encoding the large subunit of RNA polymerase I, RPA190; T and A are the sensitive and resistant types, respectively. The $\mathrm{T} \rightarrow \mathrm{A}$ change causes a tyr $\rightarrow$ phe substitution, and transforming an RPA190 allele from a resistant isolate into a sensitive strain was reported to decrease the effect of metalaxyl. Randall et al. (2014) identified a few resistant strains that lacked the T1145A SNP, which is consistent with a study that suggested that more than one major locus might determine resistance (Judelson and Roberts 1999).

The apparent identification of a metalaxyl resistance gene and reemergence of sensitive isolates suggest that a DNA-based assay for resistance may be useful for improving the management of late blight. In this paper, we demonstrate that metalaxyl can indeed be effective against the new lineages of $P$. infestans under field conditions. We also describe a high resolution melt (HRM; Vossen et al. 2009) assay for scoring the T1145A SNP genotype in RPA190. However, our results indicate that the genotype at that locus is a poor predictor of the sensitivity of isolates, and that RPA190 does not cosegregate with resistance in crosses involving some resistant isolates. Our experience with the HRM assay also indicates that 
DNA assays for diagnosing traits in $P$. infestans must address the likelihood that many strains are not simple diploids.

\section{MATERIALS AND METHODS}

Strains. Isolates of $P$. infestans were from collections at the University of California-Riverside, USDA-ARS (Corvallis, OR; a gift of N. Grünwald), or Cornell University. Assignments to clonal lineages were based on microsatellite markers, or RG57 and allozyme analysis for strains collected prior to 2011. These assays were performed as described, including the use of 11 primer sets that detect polymorphic microsatellite loci (Danies et al. 2013). Progeny were from an $8811 \times$ E13a cross $(\mathrm{A} 1 \times \mathrm{A} 2)$ described previously (Fabritius et al. 1997), and new progeny from parents (1306 and 618) that had been crossed in a prior study (Judelson et al. 1995). In the latter, oospore germination rates were $17.8 \%$. Whether oosporederived strains were parental hybrids or selfs was assessed by scoring SNPs in amplicons obtained by polymerase chain reaction (PCR) using G1 (5'-GCAAATTCTTCCTGAACGTGATCC, 5'-ACAC CACATCACCAGTCTTCAG) or G7 (5'-GAGAACCTGGAAG AAGCTTACG， 5'-GAGAACCTGGAAGAAGCTTACG) primer pairs, followed by Sanger sequencing. $G 7$ was derived from previously described marker W4 (Judelson and Roberts 1999) by in silico chromosome walking; $G 7$ is about $600 \mathrm{~kb}$ and $1 \mathrm{cM}$ away from $W 4$. About $5 \%$ of strains obtained from oospores were deemed to be nonrecombinant progeny (parental genotypes or selfs) and discarded.

Metalaxyl sensitivity assays. Strains were cultured on ryesucrose media containing metalaxyl at $0,0.5$, and $5 \mu \mathrm{g} / \mathrm{ml}$ (technical grade, $93 \%$ active ingredient) with $1.5 \%$ agar. Sensitivity was scored by comparing radial growth in the presence and absence of metalaxyl on 100-mm plates inoculated near one edge, using at least three biological replicates. Inoculum always came from cultures lacking metalaxyl. For isolates 80029, 8880, 93H3, 9026, 9086, $9400,1114,11006$, and 180, sensitivity was scored based on the increase in colony radius between 3 and 9 days after inoculation. For other strains, growth rates were determined from the slopes of linear regression lines fit to data recorded every 2 to 4 days; measurements on plates containing the drug were typically made for up to 14 days. Both types of assays yielded similar results. Resistant, intermediate, and sensitive categories were defined as $>90 \%, 40$ to $90 \%$, and $<40 \%$ growth on metalaxyl at $5.0 \mu \mathrm{g} / \mathrm{ml}$ compared with unamended media, respectively.

Field tests. Potato seed from 'Yukon Gold' and transplants of tomato 'Primo Red' were planted in Freeville, New York on 7 July 2012 and 2 August 2012, respectively. A randomized complete block design, with six blocks, was used for the experiment. Plots for each treatment (five pathogen strains, with and without fungicide) consisted of two $6 \mathrm{ft}$ rows with 12 in spacing within rows, and 3 and $5 \mathrm{ft}$ spacing between potato and tomato rows, respectively. Ridomil Gold SL (mefenoxam) was applied at 3.2 oz per acre on 21 August at 10 A.M. At 8 P.M., each plot was sprayed with $20 \mathrm{ml}$ of zoospores, which were prepared by incubating sporangia at $10^{3} / \mathrm{ml}$ for $2 \mathrm{~h}$ at $4^{\circ} \mathrm{C}$. Visual disease severity was determined after 1 and 2 weeks (27 August and 3 September 2012) using a modified version of the assessment key described by James (1971).

A general linear mixed model (JMP Pro, version 11.2.0, SAS Institute Inc., Cary, NC) was used to investigate the effect of fungicide treatment, host, isolate, and their interactions on disease severity. The response was $\log _{10}$-transformed disease severity $(\%)$. To enable log transformation of the data, a constant of 0.001 was added to all data points. The transformation was conducted to satisfy statistical assumptions for the model. Fungicide treatment, host, isolate, and their full factorial interactions were considered fixed effects. The effect of block in each experiment was treated as a random effect. Each assessment time point (7 or 14 days after infection) was analyzed separately. Noninoculated controls did not show symptoms of late blight and were therefore excluded from the analyses. The restricted maximum likelihood method was used for the mixed model. Least squares means were compared using a Tukey honestly significant difference posthoc test or test slices (JMP Pro). $P$ values from test slices were corrected for multiple comparisons using a Bonferroni correction.

RPA190 genotyping. Alleles were amplified from genomic DNA using the G1 primers listed above, resulting in a 711-bp band, or G1B primers (5'-GGCGCAAATTCTTCCTGAAC, 5' TTGCTGCTATCCACCAGAAG). The G1B primers bind internal to the G1 primers, and amplify a 280-bp band. Amplified DNA was purified and sequenced using the Sanger method. In the case of heterozygotes, peak heights in chromatograms were calculated using QSV Analyzer (Carr et al. 2009). Similar results were obtained when G1 and G1B amplicons were sequenced, and when forward and reverse sequencing primers were used. HRM assays were performed in a Bio-Rad CFX96 instrument using primers HRMU (5'-ACTTGTCGAAGATTATGACT) and HRML (5'-CCTG GCGTTTGTAGTAG). Reactions were performed in a volume of $20 \mu \mathrm{l}$ using the Bio-Rad Precision Melt Supermix kit with $10 \mathrm{ng}$ of genomic DNA, $0.2 \mu \mathrm{M}$ of each primer, and 40 cycles of $10 \mathrm{~s}$ at $95^{\circ} \mathrm{C}$, $30 \mathrm{~s}$ at $55^{\circ} \mathrm{C}$, and $30 \mathrm{~s}$ at $72^{\circ} \mathrm{C}$. Melt curves were generated using Precision Melt Analysis software.

\section{RESULTS}

Field control of US-23 and US-24 by metalaxyl. These lineages are reported to show high to intermediate sensitivities to metalaxyl, respectively, when assayed on artificial media (Danies et al. 2013). Since a premise of our research was that metalaxyl might be used to control these lineages, in concert with a DNA assay to predict isolate sensitivity, it was important to test how metalaxyl affected US-23 and US-24 in the field. Consequently, plots of potato and tomato with or without fungicide treatment were inoculated with two US-23 and two US-24 isolates and disease severity was scored after 7 and 14 days (8.27.2012 and 9.3.2012, respectively). In laboratory tests, these isolates had displayed sensitive and intermediate phenotypes. A metalaxyl-resistant strain from clonal lineage US-11 was used as a control.

In the field experiment (Fig. 1), the effectiveness of metalaxyl depended on the isolate as indicated by a significant two-way interaction between fungicide treatment and isolate $(P<0.0001$ and $P<0.02$ for the 7 - and 14-day assessments, respectively). In particular, metalaxyl was effective against US-23 and US-24 $(P<$ $0.05)$. One week after inoculation, metalaxyl suppressed disease severity caused by all four US-23 and US-24 strains by $>50 \%$, relative to their respective untreated controls. After 2 weeks, the metalaxyl treatment demonstrated a continued reduction in disease severity for both isolates of US-23 $(P<0.007)$ and one isolate of US-24 (US110157; $P<0.0001$ ) relative to the controls. In contrast, the chemical was ineffective against the US-11 strain, with no significant difference in disease severity between metalaxyltreated and untreated plants $(P>0.94)$. It is interesting to note that on artificial media this US-11 strain grows slightly faster in the presence of metalaxyl, and we also observed a trend of more growth on the fungicide-treated versus untreated plants in this field study.

Differences in host preference were also observed for US-23 and US-24. The US-23 isolates caused more symptoms on tomato than potato $(P<0.001)$ and US-24 isolates resulted in more symptoms on potato than tomato $(P<0.01)$. These differences are consistent with previous reports (Danies et al. 2013). In contrast, US-11 did not differ significantly in disease severity on the two hosts $(P>0.2)$. There was a nonsignificant three-way interaction between fungicide treatment, host, and isolate $(P=0.53$ and 0.44 for the 7 - and 14-day assessments, respectively).

Determining genotype by sequencing and HRM analysis. Since we anticipated that position 1145 of RPA190 might predict metalaxyl sensitivity, two methods were developed to genotype isolates. These were Sanger sequencing of PCR products and HRM 
analysis. Isolates found by sequencing to be A or T homozygotes or heterozygotes were distinguishable by the HRM technique (Fig. 2A, top panel). This employed primers that amplified a 63-nt region that was monomorphic in each of 22 isolates tested, not counting position 1145 .

Some heterozygous isolates seemed to be triploid or tetraploid since sequencing reactions yielded A:T ratios besides 1:1 at position 1145. This was evident when peak heights in the sequencing chromatograms were compared (Fig. 2B). For example, while the top chromatogram in the figure shows a 1:1 ratio of $\mathrm{A}$ and $\mathrm{T}$, the second chromatogram presents a 1:2 ratio, which is suggestive of triploidy. Similar results were obtained regardless of the direction of the sequencing reaction, with multiple primer sets in the polymerase
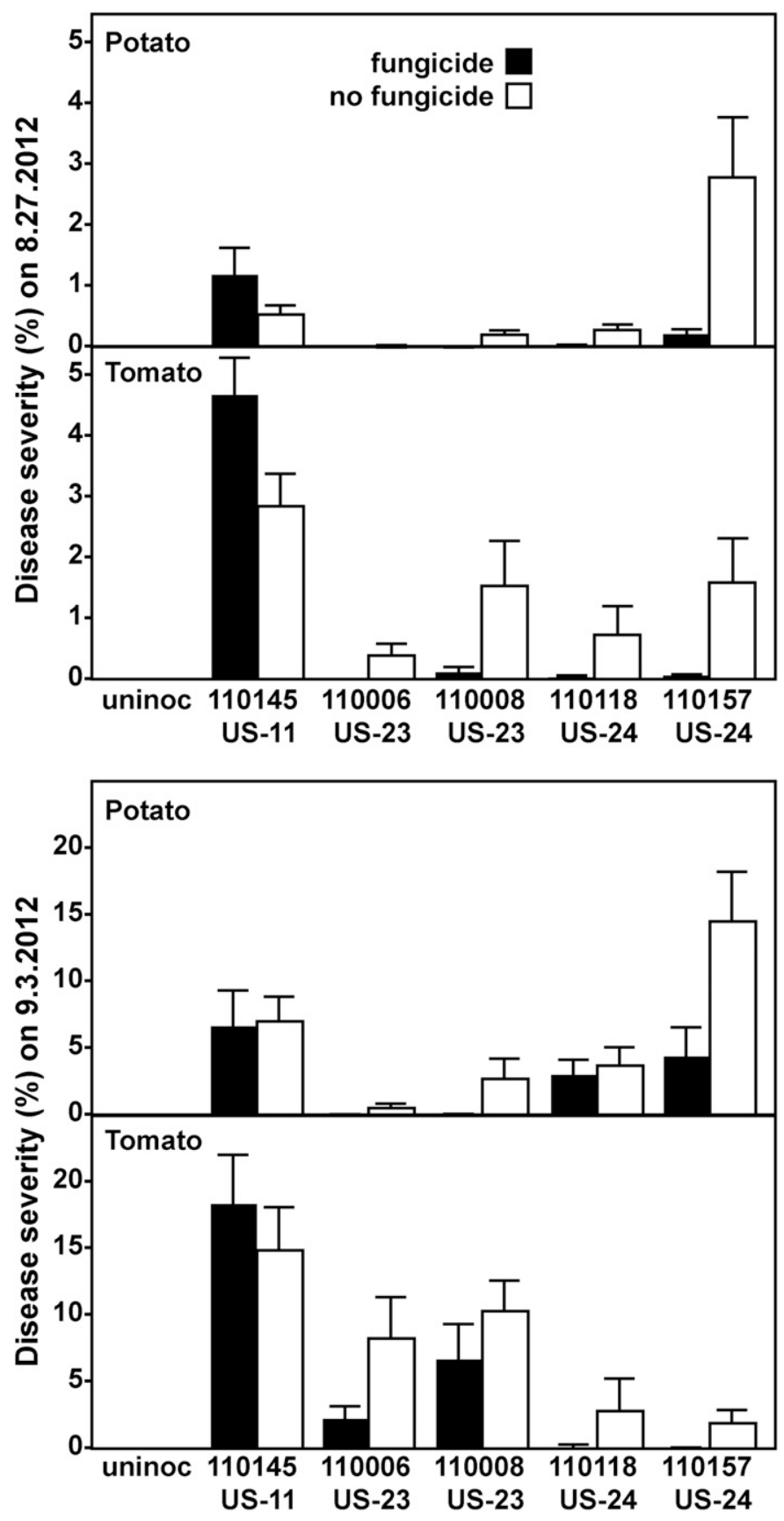

Fig. 1. Effect of metalaxyl in field assay. Plots of potato and tomato were sprayed with Ridomil Gold SL at a rate of $3.2 \mathrm{oz}$ per acre. Ten hours later, the plants were sprayed with zoospore suspensions from the indicated isolates of Phytophthora infestans. Symptoms were recorded 7 days (8.27.2012; top) and 14 days (9.3.2012; bottom) after inoculation. chain reaction $(\mathrm{PCR})$, and when isolates were passed through a zoospore (single-nucleus) purification stage. These genotypes nevertheless could still be distinguished from A or T homozygotes in the HRM assay (Fig. 2A, bottom panel).

Imperfect association of T1145A SNP with resistance. Twenty-six strains of P. infestans from North America and Europe with diverse levels of resistance were scored for the SNP by sequencing (Table 1). These included representatives of the historically prominent lineages from the United States, namely US-1, 6, 7, 8, 11, 22, 23, and 24. Most were also tested using the HRM assay, which yielded the same result as DNA sequencing.

Containing the A allele associated by Randall et al. (2014) with resistance were eight of eleven resistant isolates, four of seven intermediately resistant isolates, and none of six sensitive isolates. The exceptions included three resistant $(110145,88069$, and 8880) and three intermediate isolates $(618,1114$, and 11017) that were T homozygotes. Isolate 8880 , for example, belongs to the US-6 lineage which is one of the most metalaxyl-resistant North American genotypes (Goodwin et al. 1996), despite being homozygous for T at position 1145. Two resistant US-11 isolates varied in genotype, with isolate 110145 being homozygous for $\mathrm{T}$ and isolate 9400 an AT heterozygote. It should be noted that when isolates scored as resistant were found to lack an A allele, or when isolates recorded as sensitive contained an A allele, the genotypic and phenotypic analyses were repeated.

One explanation for these findings is that resistance in some strains is determined by genes other than RPA190. It is also possible that SNPs at other locations in RPA190 may cause resistance. We sequenced the entire 5.4-kb gene from several of the strains in Table 1 in an attempt to identify such SNPs (Supplementary Table S1), but failed to detect other SNPs associated with resistance.

Variation in US-23 and US-24 is not correlated with RPA190 genotype. Some diversity in metalaxyl sensitivity in these lineages is reported (Danies et al. 2013), and we sought to test if this could be explained by the genotype at position 1145 . Twenty isolates assigned to US-23 and 18 to US-24 by microsatellite analysis (Danies et al. 2013) were thus analyzed. Their relative growth rates in metalaxyl at $0.5 \mu \mathrm{g} / \mathrm{ml}$ compared with unamended media ranged from 11 to $95 \%$ for US-23 and 45 to $100 \%$ for US-24 (Fig. 3A and B). By comparison, strain 1306, which predates the description of metalaxyl resistance in North America, showed $<20 \%$ growth. The relative growth rates of the most highly resistant strains on metalaxyl at both 0.5 and $5.0 \mu \mathrm{g} / \mathrm{ml}$ were close to that of a US-8 isolate (Fig. 3C and D). Note that the US-23 and US-24 strains used in this experiment were selected for diversity, therefore the sensitivity distribution shown in the figure does not reflect frequencies observed in random isolates.

Genotypes obtained by DNA sequencing indicated that both the US-23 and US-24 lineages contained individuals that were T homozygotes or AT heterozygotes at position 1145. The A:T ratios of heterozygotes included $1: 1,1: 2$, and 1:3, suggesting that many were triploid or tetraploid. This did not appear to be due to heterokaryosis, since the same results were obtained when several isolates were retested after single-zoospore purification; zoospores are typically mononucleate.

Regardless of the basis of the nonstandard allele ratios, the dosage of the A allele was not strongly linked to the level of metalaxyl sensitivity. For example, AT genotypes resided at both ends of the sensitivity spectrum in US-23, and some of the more resistant US-23 and US-24 strains were TT. We considered the possibility that different results might have been obtained if testing was done at a higher concentration of metalaxyl, but this was not the case (Fig. 3C and D). A fairly good correlation was observed between relative growth on metalaxyl at 0.5 and $5 \mu \mathrm{g} / \mathrm{ml}$, with the correlation coefficient $R$ equaling 0.89 and 0.71 for US-23 and US-24, respectively.

The sequence analysis also detected three distinct genotypes in US-23 and three in US-24, when SNPs throughout the whole 711-nt 
amplicon used to score position 1145 were considered. Variation was seen at five positions including 1145, representing two and three SNPs causing synonymous and nonsynonymous substitutions, respectively (Table 2). In US-23, 11 strains were represented by genotype $23 \mathrm{~A}$, followed by 7 in $23 \mathrm{~B}$ and two in $23 \mathrm{C}$. US- 24 was less diverse, with most strains belonging to group 24A. The differences were consistent with gene conversion events rather than spontaneous mutations. For example, at positions 1338 and 1670 in US-23, the genotypes were either CC or CT, or GT or GG, respectively. There was not an obvious correlation between sensitivity level and genotype or ploidy, and the most and least sensitive strains within US-23 both belonged to group 23A.

RPA190 SNPs fail to cosegregate with resistance. The failure of the T1145A SNP to associate strongly with sensitivity in the isolates in Table 1, and in the US-23 and 24 strains in Figure 3, does not exclude the prospect that other polymorphisms in the RPA190 open reading frame play roles in resistance. The gene's regulatory sequences may also contribute to resistance by affecting RPA190 expression. To test these possibilities, we assessed whether RPA190 alleles cosegregated with resistance in controlled laboratory crosses.

First, we examined a cross between isolates 8811 (highly resistant, AT at position 1145) and E13a (sensitive, TT). The phenotypes of 24 progeny from this cross ranged from intermediate to high resistance (Fig. 4). The sensitivity levels of TT and AT progeny were not significantly different $(P=0.91$ by unpaired $t$ test). The most likely interpretation is that insensitivity is caused primarily by a locus unlinked to RPA190, which is likely homozygous in 8811.

A similar conclusion was drawn from a cross between intermediately resistant isolate 618 and sensitive strain 1306. As shown in Figure 5, the offspring ranged from low to high sensitivity. Both parents were homozygous for $\mathrm{T}$ at position 1145 , but other
TABLE 1. RNA190 genotype in 22 isolates of Phytophthora infestans

\begin{tabular}{lcccl}
\hline Isolate & Phenotype $^{\mathrm{a}}$ & 1145 $^{\text {alleles }}{ }^{\mathrm{b}}$ & A:T ratio $^{\mathrm{b}}$ & Origin and lineage $^{\mathrm{c}}$ \\
\hline 80029 & R & AA & $2: 0$ & Netherlands, 1980 \\
8811 & R & AT & $1: 1$ & United Kingdom, 1988 \\
88069 & R & TT & $0: 2$ & Netherlands, 1988 \\
8880 & R & TT & $0: 2$ & Canada, 1994 (US-6) \\
$93 \mathrm{H} 3$ & R & AT & $1: 1$ & USA, 1993 (US-7) \\
9086 & R & AT & $1: 1$ & Canada, 1994 (US-8) \\
9026 & R & AT & $1: 1$ & Canada, 1994 (US-8) \\
9400 & R & AT & $1: 2$ & Canada, 1995 (US-11) \\
110145 & R & TT & $0: 2$ & USA, 2011 (US-11) \\
11007 & R & AT & $1: 1$ & USA, 2011 (US-8) \\
1114 & I & TT & $0: 2$ & Netherlands, 1985 \\
618 & I & TT & $0: 2$ & Mexico, 1987 \\
110017 & I & TT & $0: 2$ & USA, 2011 (US-23) \\
110006 & I & AT & $1: 2$ & USA, 2011 (US-23) \\
110008 & I & AT & $1: 3$ & USA, 2011 (US-23) \\
110118 & I & AT & $1: 1$ & USA, 2011 (US-24) \\
110157 & I & AT & $1: 2$ & USA, 2011 (US-24) \\
11019 & I & AT & $1: 2$ & USA, 2011 (US-24) \\
180 & S & TT & $0: 2$ & USA (US-1) \\
510 & S & TT & $0: 2$ & Mexico, 1983 \\
550 & S & TT & $0: 2$ & Mexico, 1983 \\
1306 & S & TT & $0: 2$ & USA, 1982 \\
R0 & S & TT & $0: 2$ & USA, pre-1980 (US-1) \\
E13a & S & TT & $0: 2$ & Egypt, 1984 \\
11016 & S & TT & $0: 2$ & USA, 2011 (US-22) \\
11006 & S & TT & $0: 2$ & USA, 2011 (US-1) \\
\hline
\end{tabular}

a Resistant (R), intermediate (I), and sensitive (S) are defined as $>90 \%, 40$ to $90 \%$, and $<40 \%$ growth on metalaxyl at $5.0 \mu \mathrm{g} / \mathrm{ml}$ compared with unamended media.

b Based on Sanger sequencing of RPA190 gene. Allele ratios determined using QSV Analyzer are shown to the nearest integer. When a homozygous state was inferred, the strain is scored as a diploid (0:2 or 2:0), although some could be of higher ploidy.

c Genotypes matching common "US" clonal lineages are indicated in parentheses. The date of isolation of some strains are unknown.
A

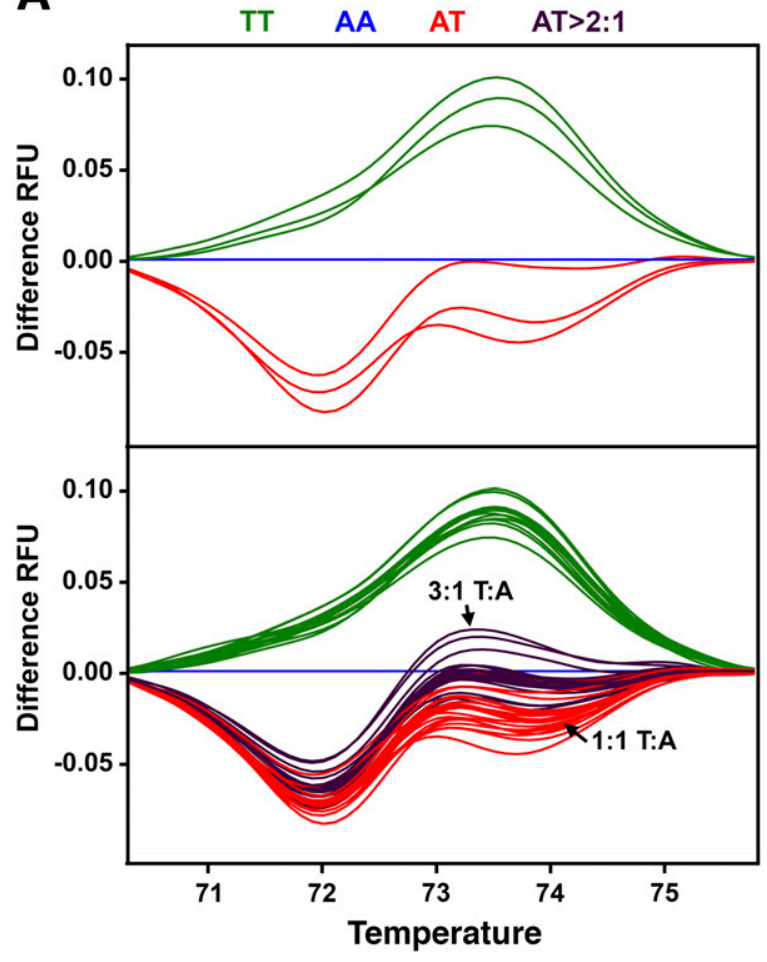

B
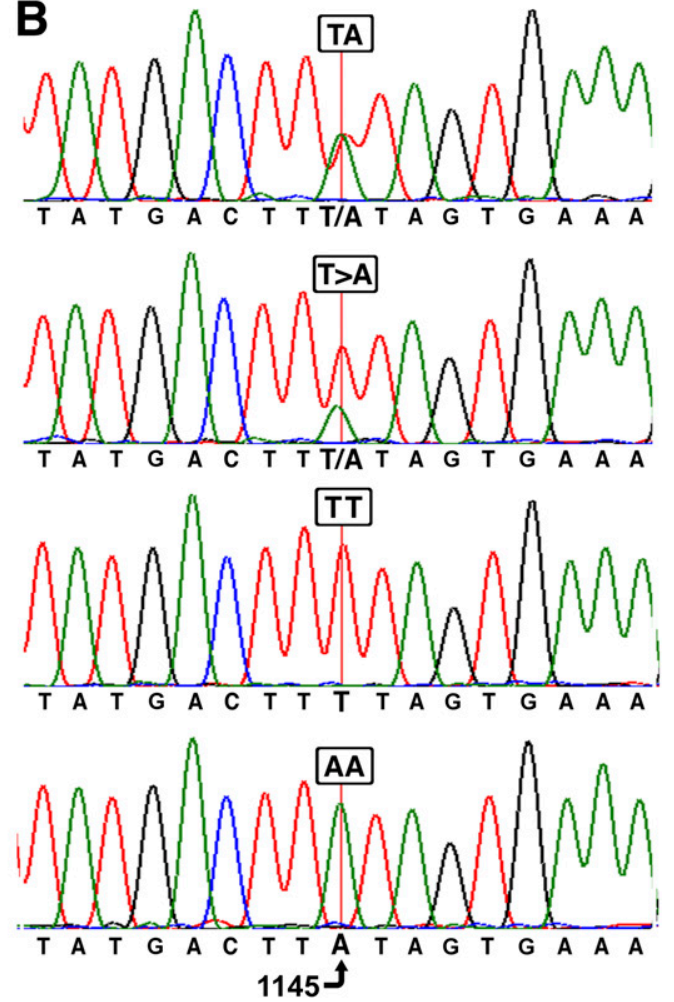

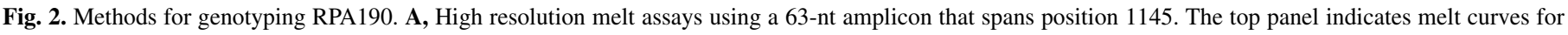

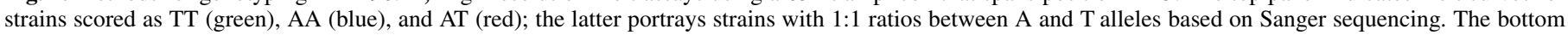

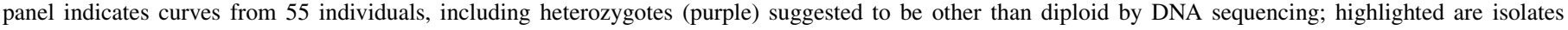

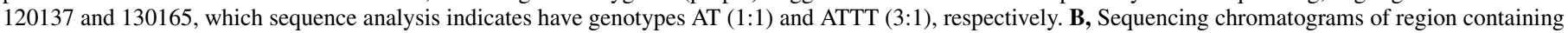

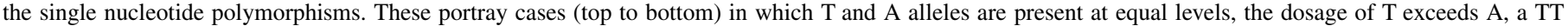
homozygote, and an AA homozygote. 
polymorphisms were present which enabled us to assess whether RPA190 cosegregated with metalaxyl resistance. Their analysis indicated that the sensitivity of progeny inheriting the two alternate alleles of RPA190 from 618 were not significantly different $(P=$ 0.92 by unpaired $t$ test). An equivalent result was obtained by treating the 25 most resistant and 25 sensitive progeny as separate classes, in which case RPA190 and resistance were unlinked with a recombination fraction of 0.50 . The most likely interpretation is that insensitivity is determined mainly by a locus unlinked to RPA190, which is likely heterozygous in 618. The locus determining insensitivity is however genetically linked to marker $G 7$, which was obtained by in silico chromosome walking from marker $W 4$, which was identified previously by screening for random amplified polymorphic DNA markers associated with resistance (Judelson and Roberts 1999). Treating the 25 most resistant and 25 sensitive progeny as separate classes, $G 7$ had a recombination frequency of $0.10(\mathrm{LOD}=8)$ with the resistance determinant.

\section{DISCUSSION}

The use of site-specific fungicides has expanded over past decades, since many have systemic and curative activity. Their effectiveness relative to traditional multisite inhibitors has been countered by the increased likelihood that resistance will arise. The

TABLE 2. Subclasses within lineages based on RPA190 single nucleotide polymorphisms

\begin{tabular}{lccccccc}
\hline & & \multicolumn{7}{c}{ Position in gene } & \\
\cline { 3 - 6 } Lineage & Subclass & 1145 & 1338 & 1371 & 1670 & 1693 & Number of strains \\
\hline US-23 & 23A & AT & CC & TT & GG & CT & 11 \\
& 23B & AT & CT & TT & GT & CT & 7 \\
& 23C & TT & CT & TT & GT & CC & 2 \\
US-24 & 24A & AT & CT & CT & GT & CC & 16 \\
& 24B & AT & CC & CT & GG & CC & 1 \\
& 24C & TT & CT & TT & GT & CC & 1 \\
\hline
\end{tabular}

B (US-24)

$\%$ growth on $0.5 \mu \mathrm{g} / \mathrm{ml}$ met
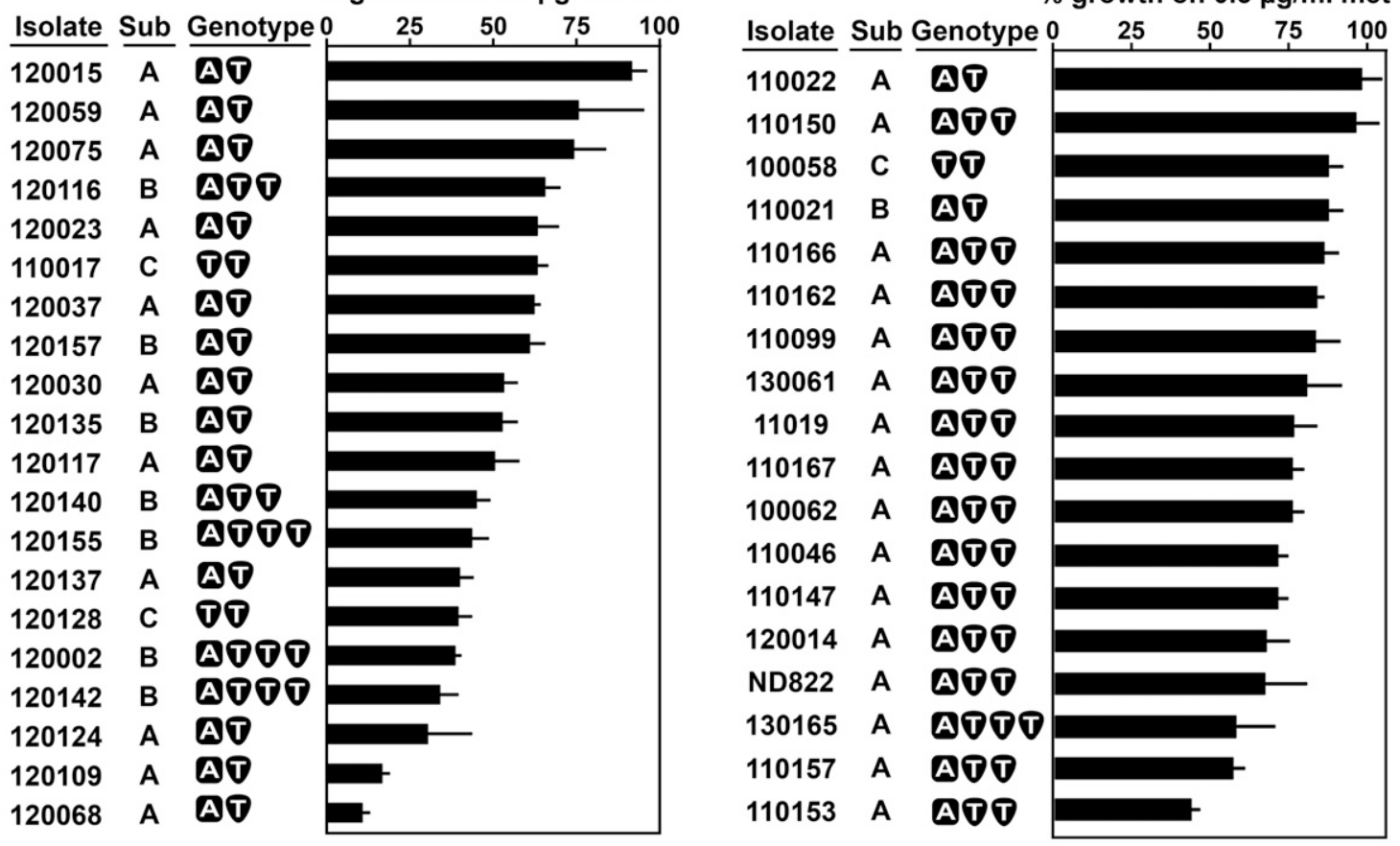

D (US-24)

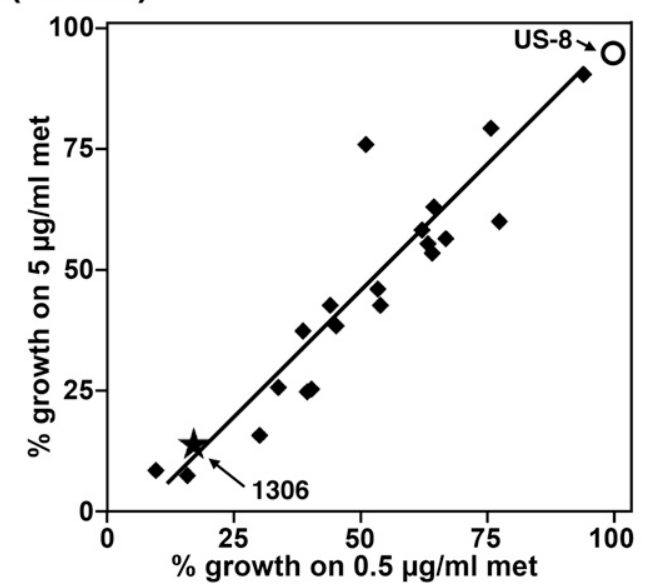

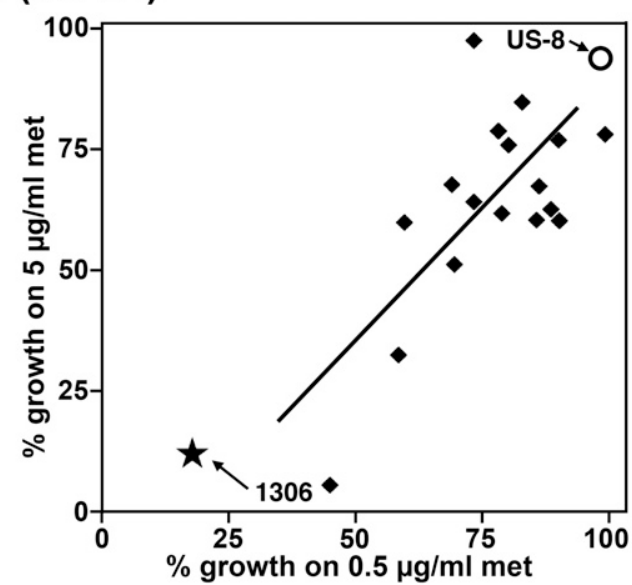

Fig. 3. Characteristics of US- 23 and US-24 isolates. A, US-23. Indicated are assignments to genotype subgroups A, B, or C as defined in Table 2 (Sub), genotypes at position 1145 with the number of A and T symbols reflecting ratios from QSV Analyzer rounded to the nearest integer, and relative growth on metalaxyl at $0.5 \mu \mathrm{g} / \mathrm{ml}$ (met) in rye-sucrose media compared with unamended media. B, same as panel A but for US- 24 . C, Relative growth of US- 23 strains on metalaxyl at 0.5 and $5 \mu \mathrm{g} / \mathrm{ml}$ compared with unamended media. Included for comparison are sensitive isolate 1306 (star) and resistant US-8 isolate 110007 (circle). D, Same as panel C but with US-24. 
study by Randall et al. (2014) was a landmark since it revealed a genetic basis of resistance in $P$. infestans to metalaxyl, which has been an important tool against oomycetes. Our work however failed to connect that genetic change with the sensitivity level of many isolates, including lineages now prevalent in the United States. The gene encoding the RNA polymerase subunit implicated by Randall et al. (2014) also did not cosegregate with resistance in our crosses involving insensitive isolates from Mexico and the United Kingdom. While we successfully developed an assay for rapid RPA190 genotyping using the HRM technique, such tests will not be useful for informing management practices until more is learned about the basis of resistance.

That multiple genes may determine metalaxyl resistance is not surprising, since it behaves genetically as a complex trait. This was illustrated by our $618 \times 1306$ cross, where offspring displayed a continuous distribution of sensitivities. Genes that potentially affect resistance include those that encode the structural target of metalaxyl, inactivating enzymes, and efflux proteins. The latter include $\mathrm{ABC}$ transporters, which also contribute to resistance against chemistries used against true fungi (Abou Ammar et al. 2013; Omrane et al. 2015 ) and were observed to be upregulated in strains of $P$. infestans adapted to growth on metalaxyl (Childers et al. 2014). Since RNA polymerase $\mathrm{I}$ is a multiprotein complex, alterations in more than one subunit might also influence sensitivity. The T1145A SNP changes

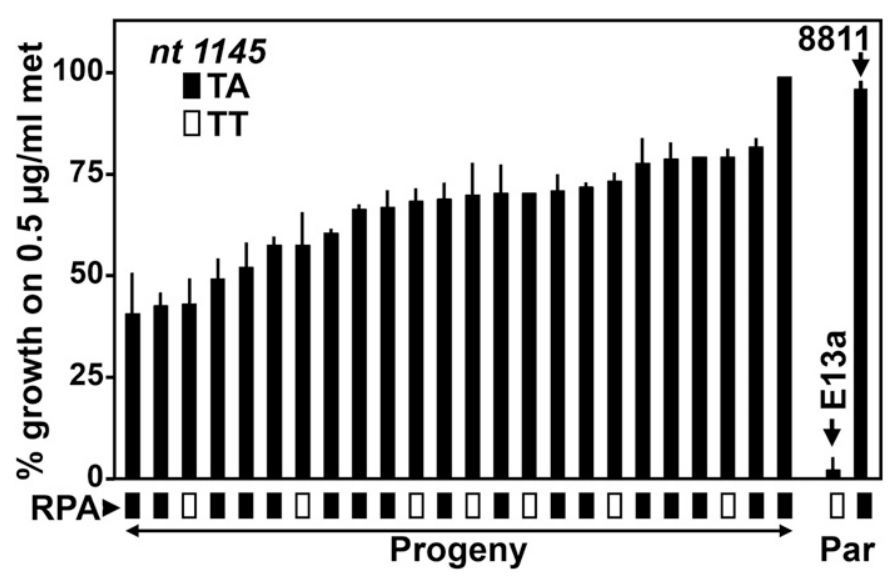

Fig. 4. Metalaxyl resistance and 1145 genotype of progeny from $8811 \times \mathrm{E} 13 \mathrm{a}$ cross. Shown is the relative growth of the strains on metalaxyl at $0.5 \mu \mathrm{g} / \mathrm{ml}$ compared with unamended media, and the genotype of position 1145 of RPA190. Closed and open boxes beneath the graph represent TA and TT genotypes, respectively. an amino acid in the RPA190 clamp domain, through which rRNA exits the polymerase. Based on the structure of yeast RNA polymerase I holoenzyme, the protrusion domain of RPA135 resides near the clamp (Engel et al. 2013; Fernandez-Tornero et al. 2013). Consequently, changes in both RPA135 and RPA190 might influence the response to metalaxyl.

While our sequence analysis of RPA190 in US-23 and US-24 was not informative for diagnosing metalaxyl resistance, it revealed diversity in the lineages. These lineages, which are defined by an 11-locus microsatellite genotype, are believed to reproduce asexually although changes can occur through spontaneous mutation and gene conversion. Nevertheless, we were surprised to detect three distinct genotypes based on just a short stretch of a single gene, in addition to potential variation in ploidy. The US-23 and US24 lineages were first detected in 2009, and the pace at which their sublineages arose can be estimated knowing that the isolates studied here were collected between 2010 and 2013. There are also precedents for variation in other lineages of $P$. infestans based on virulence and molecular markers (Abu-El Samen et al. 2003a, b; Goodwin et al. 1995; Harbaoui et al. 2014). Variation in the level of metalaxyl sensitivity has also been reported in other lineages, such as US-8 and US-11 (Daayf and Platt 2002).

Our detection of nonstandard allele ratios within US-23 and US24 is consistent with prior information of the diversity of nuclear states in $P$. infestans. Isolates are reported to include $2 \mathrm{n}, 3 \mathrm{n}, 4 \mathrm{n}$, and aneuploid types (Catal et al. 2010; Ritch and Daggett 1995; Tooley and Therrien 1987). US-24 isolates in particular were observed to contain a three-allele (100/100/111) allozyme genotype for glucose6-phosphate isomerase, which suggests that lineage is not diploid (Hu et al. 2012). Ploidy levels are also known to vary between nuclei within a single $P$. infestans culture (Catal et al. 2010). After passing through a spore stage, this would give rise to a population of individuals with a range of nuclear contents, and this may explain the variation within US-23 and US-24.

That ploidy varies within $P$. infestans must be factored in when designing DNA assays for predicting traits. There is growing interest, both with $P$. infestans and other phytopathogens, in using DNA assays to diagnose traits such as fungicide resistance, virulence (race), and mating type (Carpezat et al. 2014; Derevnina and Michelmore 2015; Dufour et al. 2011; Short et al. 2014). Our experience with $P$. infestans shows that consideration must be given to the phenotype that results from all possible allele dosages, since strains having 3:1, 2:1, and 1:1 ratios at a given locus may have distinct phenotypes. Quantitative rather than qualitative DNA assays may therefore be required. Nucleic acid sequencing should be sufficient, but has a slower turn-around time than other technologies. Our HRM assay is faster than sequencing, and could distinguish AT

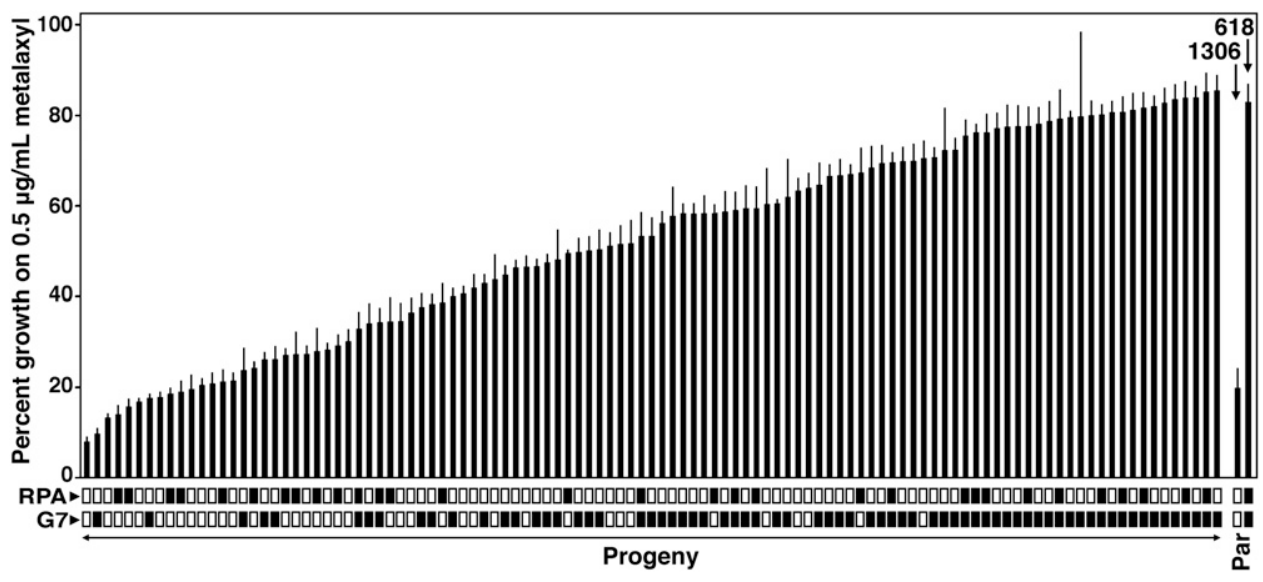

Fig. 5. Metalaxyl resistance and genotypes of $618 \times 1306$ cross. Shown is the relative growth of the strains on metalaxyl at $0.5 \mu \mathrm{g} / \mathrm{ml}$ compared with unamended media, and the genotype of position 1145 of RPA190 and an unlinked marker, G7. Closed and open boxes beneath the graph represent TA and TT genotypes for RPA190, and CT and TT for locus G7, respectively. 
heterozygotes with different ratios from AA and TT homozygotes within RPA190, but this approach might not succeed with other loci. The complication of diversity in ploidy is not unique to $P$. infestans. It has been described in other species of Phytophthora (Bertier et al. 2013), in ascomycetes and basidiomycetes (Albertin and Marullo 2012), in nematodes (Triantaphyllou 1991), and is suspected in Pythium (Spies et al. 2011).

\section{ACKNOWLEDGMENTS}

This work was supported by grant 2011-68004-30154 from USDANIFA. We thank members of the USAblight team for providing many of the isolates used in this study. We are especially grateful to S. Whisson for generously sharing unpublished data. We also thank K. Myers for helping to maintain and ship isolates.

\section{LITERATURE CITED}

Abou Ammar, G., Tryono, R., Doll, K., Karlovsky, P., Deising, H. B., and Wirsel, S. G. R. 2013. Identification of ABC transporter genes of Fusarium graminearum with roles in azole tolerance and/or virulence. PLoS One 8:e79042.

Abu-El Samen, F. M., Secor, G. A., and Gudmestad, N. C. 2003a. Variability in virulence among asexual progenies of Phytophthora infestans. Phytopathology 93:293-304.

Abu-El Samen, F. M., Secor, G. A., and Gudmestad, N. C. 2003b. Genetic variation among asexual progeny of Phytophthora infestans detected with RAPD and AFLP markers. Plant Pathol. 52:314-325.

Albertin, W., and Marullo, P. 2012. Polyploidy in fungi: Evolution after wholegenome duplication. Proc. R. Soc. B 279:2497-2509.

Bertier, L., Leus, L., D'hondt, L., de Cock, A. W. A. M., and Hofte, M. 2013. Host adaptation and speciation through hybridization and polyploidy in Phytophthora. PLoS One 8:e85385.

Carpezat, J., Bothorel, S., Daverdin, G., Balesdent, M. H., and Leflon, M. 2014. Use of high resolution melting analysis to genotype the avirulence avrLM4-7 gene of Leptosphaeria maculans, a fungal pathogen of Brassica napus. Ann. Appl. Biol. 164:430-440.

Carr, I. M., Robinson, J. I., Dimitriou, R., Markham, A. F., Morgan, A. W., and Bonthron, D. T. 2009. Inferring relative proportions of DNA variants from sequencing electropherograms. Bioinformatics 25:3244-3250.

Catal, M., King, L., Tumbalam, P., Wiriyajitsomboon, P., Kirk, W. W., and Adams, G. C. 2010. Heterokaryotic nuclear conditions and a heterogeneous nuclear population are observed by flow cytometry in Phytophthora infestans. Cytometry A 77A:769-775.

Childers, R., Danies, G., Myers, K. L., Fei, Z., Small, I. M., and Fry, W. 2014. Acquired resistance to mefenoxam in sensitive isolates of Phytophthora infestans. Phytopathology 105:342-349.

Crute, I. R., and Harrison, J. M. 1988. Studies on the inheritance of resistance to metalaxyl in Bremia lactucae and on the stability and fitness of field isolates. Plant Pathol. 37:231-250.

Daayf, F., and Platt, H. W. 2002. Variability in responses of US-8 and US-11 genotypes of potato and tomato isolates of Phytophthora infestans to commercial fungicides in vitro. Am. J. Potato Res. 79:433-441.

Danies, G., Small, I. M., Myers, K., Childers, R., and Fry, W. E. 2013. Phenotypic characterization of recent clonal lineages of Phytophthora infestans in the United States. Plant Dis. 97:873-881.

Davidse, L. C., Gerritsma, O. C. M., Ideler, J., Pie, K., and Velthuis, G. C. M. 1988. Antifungal modes of action of metalaxyl, cyprofuram, benalaxyl and oxadixyl in phenylamide-sensitive and phenylamide-resistant strains of Phytophthora megasperma f. sp. medicaginis and Phytophthora infestans. Crop Prot. 7:347-355.

Davidse, L. C., Looijen, D., Turkensteen, L. J., and Vanderwal, D. 1981. Occurrence of metalaxyl-resistant strains of Phytophthora infestans in Dutch potato fields. Neth. J. Plant Pathol. 87:65-68.

Derevnina, L., and Michelmore, R. W. 2015. Wheat rusts never sleep but neither do sequencers: Will pathogenomics transform the way plant diseases are managed? Genome Biol. 16:44.

Diriwachter, G., Sozzi, D., Ney, C., and Staub, T. 1987. Cross-resistance in Phytophthora infestans and Plasmopara viticola against different phenylamides and unrelated fungicides. Crop Prot. 6:250-255.

Dufour, M. C., Fontaine, S., Montarry, J., and Corio-Costet, M. F. 2011. Assessment of fungicide resistance and pathogen diversity in Erysiphe necator using quantitative real-time PCR assays. Pest Manag. Sci. 67:60-69.

Engel, C., Sainsbury, S., Cheung, A. C., Kostrewa, D., and Cramer, P. 2013. RNA polymerase I structure and transcription regulation. Nature 502:650-655.
Fabritius, A.-L., Shattock, R. C., and Judelson, H. S. 1997. Genetic analysis of metalaxyl insensitivity loci in Phytophthora infestans using linked DNA markers. Phytopathology 87:1034-1040.

Fernandez-Tornero, C., Moreno-Morcillo, M., Rashid, U. J., Taylor, N. M. I., Ruiz, F. M., Gruene, T., Legrand, P., Steuerwald, U., and Muller, C. W. 2013. Crystal structure of the 14-subunit RNA polymerase I. Nature 502: 644-649.

Goodwin, S. B., Smart, C. D., Sandrock, R. W., Deahl, K. L., Punja, Z. K., and Fry, W. E. 1998. Genetic charge within populations of Phytophthora infestans in the United States and Canada during 1994 to 1996: Role of migration and recombination. Phytopathology 88:939-949.

Goodwin, S. B., Sujkowski, L. S., and Fry, W. E. 1995. Rapid evolution of pathogenicity within clonal lineages of the potato late blight disease fungus. Phytopathology 85:669-676.

Goodwin, S. B., Sujkowski, L. S., and Fry, W. E. 1996. Widespread distribution and probable origin of resistance to metalaxyl in clonal genotypes of Phytophthora infestans in the United States and western Canada. Phytopathology 86:793-800.

Grasso, V., Palermo, S., Sierotzki, H., Garibaldi, A., and Gisi, U. 2006. Cytochrome $\mathrm{b}$ gene structure and consequences tor resistance to Qo inhibitor fungicides in plant pathogens. Pest Manag. Sci. 62:465-472.

Harbaoui, K., Hamada, W., Li, Y., Vleeshouwers, V. G. A. A., and van der Lee, T. 2014. Increased difficulties to control late blight in Tunisia are caused by a genetically diverse Phytophthora infestans population next to the clonal lineage NA-01. Plant Dis. 98:898-908.

Hu, C. H., Perez, F. G., Donahoo, R., McLeod, A., Myers, K., Ivors, K., Secor, G., Roberts, P. D., Deahl, K. L., Fry, W. E., and Ristaino, J. B. 2012. Recent genotypes of Phytophthora infestans in the eastern United States reveal clonal populations and reappearance of mefenoxam sensitivity. Plant Dis. 96:1323-1330.

James, W. C. 1971. An illustrated series of assessment keys for plant diseases, their preparation and usage. Can. Plant Dis. Surv. 51:39-65.

Judelson, H. S., and Roberts, S. 1999. Multiple loci determining insensitivity to phenylamide fungicides in Phytophthora infestans. Phytopathology 89: 754-760.

Judelson, H. S., Spielman, L. J., and Shattock, R. C. 1995. Genetic-mapping and non-Mendelian segregation of mating-type loci in the oomycete, Phytophthora infestans. Genetics 141:503-512.

Lee, T. Y., Mizubuti, E., and Fry, W. E. 1999. Genetics of metalaxyl resistance in Phytophthora infestans. Fungal Genet. Biol. 26:118-130.

Omrane, S., Sghyer, H., Audeon, C., Lanen, C., Duplaix, C., Walker, A. S., and Fillinger, S. 2015. Fungicide efflux and the MgMFS1 transporter contribute to the multidrug resistance phenotype in Zymoseptoria tritici field isolates. Environ. Microbiol. 17:2805-2823.

Proffer, T. J., Berardi, R., Ma, Z. H., Nugent, J. E., Ehret, G. R., McManus, P. S., Jones, A. L., and Sundin, G. W. 2006. Occurrence, distribution, and polymerase chain reaction-based detection of resistance to sterol demethylation inhibitor fungicides in populations of Blumeriella jaapii in Michigan. Phytopathology 96:709-717.

Randall, E., Young, V., Sierotzki, H., Scalliet, G., Birch, P. R. J., Cooke, D. E. L., Csukai, M., and Whisson, S. C. 2014. Sequence diversity in the large subunit of RNA polymerase I contributes to mefenoxam insensitivity in Phytophthora infestans. Mol. Plant Pathol. 15:664-676.

Ritch, D. L., and Daggett, S. S. 1995. Nuclear-DNA content and chromosomenumber in German isolates of Phytophthora infestans. Mycologia 87:579-581.

Shattock, R. C. 1988. Studies on the inheritance of resistance to metalaxyl in Phytophthora infestans. Plant Pathol. 37:4-11.

Short, D. P. G., Gurung, S., Maruthachalam, K., Atallah, Z. K., and Subbarao, K. V. 2014. Verticillium dahliae race 2-specific PCR reveals a high frequency of race 2 strains in commercial spinach seed lots and delineates race structure. Phytopathology 104:779-785.

Spies, C. F. J., Mazzola, M., Botha, W. J., Langenhoven, S. D., Mostert, L., and Mcleod, A. 2011. Molecular analyses of Pythium irregulare isolates from grapevines in South Africa suggest a single variable species. Fungal Biol. 115:1210-1224.

Taylor, R. J., Salas, B., Secor, G. A., Rivera, V., and Gudmestad, N. C. 2002. Sensitivity of north american isolates of Phytophthora erythroseptica and Pythium ultimum to mefenoxam (metalaxyl). Plant Dis. 86:797-802.

Tooley, P. W., and Therrien, C. D. 1987. Cytophotometric determination of the nuclear DNA content of 23 Mexican and 18 non-Mexican isolates of Phytophthora infestans. Exp. Mycol. 11:19-26.

Triantaphyllou, A. C. 1991. Further-studies on the role of polyploidy in the evolution of Meloidogyne. J. Nematol. 23:249-253.

Vossen, R. H., Aten, E., Roos, A., and den Dunnen, J. T. 2009. High-resolution melting analysis (HRMA): More than just sequence variant screening. Hum. Mutat. 30:860-866. 